\title{
Olympic lifting vs. traditional lifting methods for North American high school football players
}

\author{
Matt Roberts, Mark DeBeliso
}

Department of Kinesiology and Outdoor Recreation, Southern Utah University, Cedar City, UT, USA.

\begin{abstract}
A stronger emphasis has been placed on increasing strength, power, size, and speed for today's football player. The key to obtaining these abilities and skills are accomplished in the weight room. There are various types of lifting methods when it comes to resistance training (RT) for football. Two types of lifting styles that are often used for high school football weight training programs are the traditional lifting or core training programs and Olympic style lifting. It is thought that Olympic lifts help to increase power, which is something that can be transferred onto the playing field and help improve overall performance. This study compared two different styles of RT (traditional vs. Olympic) in North American High School football athletes in order to determine if one RT protocol was superior at improving measures of speed, strength, and power. Forty male, high school football players ranging from 14 to 18 years old participated in an eight week, off-season, early morning football resistance training program. Each participant was randomly selected into either the traditional resistance protocol (TR: $n=20$ ) or Four Quarters protocol (FQ: $n=20$ ) experimental groups. The players each had the same weight lifting/conditioning routine, however, twice a week the FQ group performed an Olympic lift variation and the TR group performed a dead lift. Pre- and post-assessment scores were obtained and compared in the 1-RM squat, 1-RM power clean, vertical jump (VJ) and 9.14 meter sprint. Pre- postscores were compared with in each group with a dependent t-test. A gain score was also calculated for each dependent variable (post-pre score) and compared between the experimental groups with an independent t-tests $(\mathrm{p} \leq 0.05)$. Both groups made improvements in pre to post assessments in the 1-RM squat, 1-RM power clean, and VJ $(\mathrm{p}<0.05)$. The gain scores for the 1-RM squat, 1-RM power clean were significantly greater for the FQ group $(\mathrm{p}<0.05)$. Neither group improved 9.14 meter sprint times $(\mathrm{p}>0.05)$. The results of this study are consistent with those found in previous literature demonstrating that increases in both the 1-RM squat and 1$\mathrm{RM}$ power clean are better achieved using an Olympic lift
\end{abstract}

variations when compared to using a traditional lifting method. Resistance training programs for athletes are designed with the goal of improving physical performance on the field of play. The results of this study suggest that improvements in muscular strength and power as measured by the 1-RM squat and 1-RM power clean are best achieved with a RT protocol that includes the Olympic lift variation of the power clean.

Keywords. High school athletes, Olympic lifting, power, strength, traditional lifting.

\section{Introduction}

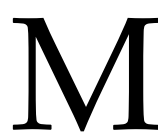

ore than ever before a stronger emphasis has been placed on increasing strength, power, size, and speed for today's athlete. In sports, particularly North American football, power is the key to many athletic performances (Garhammer, 1993). Athletic success is mainly determined by the ability of an athlete to produce powerful movements (Haff et al., 2001). By fostering and improving an athlete's power output capabilities, this will give them the advantage over their opponents and ultimately lead them to success (Haff et al., 2001).

Explosive movements and power are important on all competitive levels, but is especially evident in the National Football League (NFL). Players being drafted to professional football leagues have shown how important it is to be bigger, faster, and stronger. These players are not only selected based on their physical stature, but also on their physical abilities, and are tested on such during the NFL combine. In the 2013 NFL draft two players were selected in the first round based on their size and speed. The $5^{\text {th }}$ overall pick,

Received: July 2, 2018 - Accepted: August 31, 2018 - Published: September 20, $2018 \quad \square$ M. DeBeliso, e-mail: markdebeliso@suu.edu To cite this article: Roberts M, DeBeliso M. Olympic lifting vs. traditional lifting methods for North American high school football players. Turk J Kinesiol, 2018; 4(3): 91-100. DOI: 10.31459/turkjkin.439870 
Ziggy Ansah, was drafted by the Detroit Lions even though he only had three years of football experience and only one season as a starter. However, Ziggy got his start as a sprinter and was able to achieve phenomenal times in short distances, which demonstrated his pure power potential. Due to his incredible power he was not only drafted, but is now playing in the NFL with great success. Another example of an athlete that has superior power output is Von Miller, a linebacker for the Denver Broncos. This was evident during the playoffs in 2016 as he dominated his opponents on the field. During the Super Bowl alone he had 2.5 sacks, 6 tackles, 2 quarterback hits, 1 deflected pass, and 2 forced fumbles (Patra, 2016). This ultimately led his team to a Super Bowl victory and being awarded Super Bowl MVP. His physical skill set and abilities demonstrate how a power athlete at his professional level not only possesses the speed to cover wide receivers downfield in pass coverage, but he also has the power and strength to run over offensive linemen to get to the quarterback. The NFL values this type of power output from an athlete so much that after his Super Bowl performance, the Denver Bronco's negotiated a contract making him the highest paid defensive player in the NFL (Greenberg, 2016).

No specific playing level seems to be exempt from trying to obtain these abilities and skills, and even high school football athletes and teams are constantly working at becoming bigger, faster, and stronger. A recent survey of Utah high school football strength and conditioning coaches found that $91 \%$ of coaches use Olympic lifting in their resistance training programs (Weaver \& DeBeliso, 2015). It has been shown that those teams that utilize a more modern power oriented lifting program achieve better gains and are bigger, faster, and stronger than their competition, which typically equates to more successful football programs. In 2011, a survey of 108 head football coaches from 42 states with championship programs accredited their off-season weight lifting and speed programs as one of the top 10 reasons for their success (Fore, 2013).

When trying to improve performance, training and activity such as weight-lifting, sprinting, and vertical jump would drastically improve one's overall power production leading to an increased performance (Enoka, 2002). It's no secret that the key to obtaining these abilities and skills is through work done in the weight room. A number of high school, college, and professional football programs throughout the country spend a vast amount of their time in the weight room. However, each program differs in philosophy and methodology. It has been said that these athletes, particularly high school athletes, will show improvements no matter what they do as long as they work out in the weight room. One can't help but wonder if there is a specific training program that is more beneficial than the others.

There are many different ways to train in the weight room. Typically traditional lifting has been a major focus for a number of weight lifting programs. This type of training is popular because it has been found to yield gains in overall strength (Ebben \& Blackard, 1997; McBride et al., 1999). Traditional lifting involves lifts such as benching, squatting, and dead lifts. A lot of veteran coaches agree with this type of program due to its simplicity and easiness of application and supervision. However, this type of thinking can minimize the gains athletes can achieve in the weight room. For example using a dead lifts and squat variations fail to develop the explosive qualities needed to perform successfully in many athletic performances, especially North American football. Olympic lifting variations utilize the triple extension, which is the key to athletic power (Frounfelter, 2009; Channell \& Barfield, 2008). During the triple extension the ankles, knees, and hips fully extend to generate a powerful movement (Frounfelter, 2009; Hendrick \& Wada, 2008). Similar movements can be found on the football field when performing skills such as tackling another player or jumping to catch a pass, and because of this it is believed that specific Olympic weight lifting variations can be transferred to sports specific skills (Hendrick \& Wada, 2008). However, if an athlete is unable to generate power, their abilities will be limited, thereby, ultimately affecting their performance (Arnheim \& Prentice, 2000). Due to this need for the development of the ability to generate high muscular power output, a more modern lifting style has turned its focus to the implementation of Olympic style lifts (and derivatives) such as the power clean, hang clean, and snatch. Olympic lifts have been found to elicit more power output (Ebben \& Blackard, 1997; McBride et al., 1999; Hoffman et al., 2004; Hackett et al., 2016). Today's football training programs are moving towards incorporating this modern approach of Olympic lifts because these lifts focus on the explosive muscular power generated by the triple extension, 
which presumably transfers to the skills or abilities needed on the football field. A study performed by Burger, Boyer-Kendrick \& Dolny (2000) compared traditional lifting to complex lifting, which included both Olympic lifts and plyometrics, and found that while both groups demonstrated improvements, the complex group showed more significant improvements in power output compared to the traditional group. The National Strength and Conditioning Association (NSCA) and American College of Sports Medicine (ACSM) also promotes the use of Olympic style lifts in order to optimize athletic performance (Bruce-Low \& Smith, 2007). In North American college football athletes, the use of Olympic lifting has been found to be more advantageous in both strength and power development (Hoffman et al., 2004). For the purpose of this paper, Olympic lifting refers to the clean and jerk, the snatch, as well as the derivatives of these movements. Olympic lifting derivatives are variations of the snatch as well as the clean and jerk that also require triple extension, but that eliminate portions of the full movements (i.e. snatch, clean and jerk) (Sanders et al., 2017).

Most studies have focused on the use of Olympic lifts with collegiate or professional athletes, but there is limited research conducted with high school athletes or adolescents. However, one study specifically found Olympic lifting improved performance in speed and agility in children (Chaouachi et al., 2013). Additional studies by Harbili \& Alptekin (2014), Small et al. (2008), Faigenbaum et al. (2007), and Faigenbaum et al. (1996) established that children and adolescents that participate in resistance training programs have greater strength and power than those children and adolescents that do not. A well designed resistance training program for children and adolescents has been found to increase strength by $30-50 \%$ in $8-12$ weeks (Dahab \& McCambridge, 2009). However, another study found that an adolescent traditional resistance training program has demonstrated improvements in skill-related fitness (Warning et al., 2016). The studies mentioned above demonstrate that there are inconsistencies in the literature and in the research as to what type of resistance training programs should be used with adolescents and children to obtain the greatest strength and power gains.

Due to the lack of research and inconsistencies found in what type of resistance training program is most beneficial, the purpose of the study is to compare one aspect of the Olympic style resistance training (RT) program to a more traditional method.

The main aspect of this study focuses on the implementation of an Olympic style-lifting program, called the Four Quarters workout (a hang clean progression workout). It is designed to obtain optimal gains in speed, strength and power through the utilization of the triple extension that is achieved during the execution of Olympic lift derivatives. The study will compare the speed, strength and power gains of individuals in the Four Quarters RT program to those in a traditional lifting program (TR) through evaluation of a 1-RM power clean, 1-RM back squat, vertical jump (VJ), and $9.14 \mathrm{~m}$ sprint.

It was hypothesized that the utilization of the Four Quarters RT would yield greater improvements in the 1-RM back squat (BS), 1-RM power clean (PC), VJ, and 9.14 meter sprint than a TR protocol in a population of North American High School football players.

\section{Methods}

\section{Participants}

This study consisted of 40 male high school football players ranging in age from 14 to 18 years old. Each of these participants were players for the North Sanpete High School football team located in Mt. Pleasant, Utah. This study was conducted in the winter and spring months of 2014 as part of their off-season, early morning football resistance training program. Each participant was randomly selected into two groups, the TR $(n=20)$ or FQ $(n=20)$ lifting groups, based off of their pre-assessments in the PC and BS 1-RM. Participants had previous exposure and were very familiar with both weight room and power field assessment procedures.

Permission from the Institutional Review Board and Human Subject approval was obtained before conducting any training or assessments of athletes. Also informed written consent from the parents and assent from the children was obtained before any action in the study took place. Each participant and parent was made fully aware that participation in this study was completely voluntary and they had the right to withdraw from this study at any time and for any reason. All participants were properly instructed and supervised throughout the entirety of the study including assessments and programmed strength and 
conditioning routines. The study administrators were in attendance during all training and evaluation sessions, and were very familiar with the equipment and instruments that were used.

\section{Instruments and Apparatus}

The instrumentation and equipment used for this study included the assessment of strength and power by using the Elite platform with both standard Olympic barbell and bumper plates, and the bar being a standard $20.45 \mathrm{~kg}$ Olympic bar for the 1-RM PC and BS. Also the instrumentation and equipment for the field assessments included the following: measuring tape and Polaris Sports Timing System for assessment of the 9.14 meter sprint and the Probotics Just Jump system for assessment of the VJ. All of the equipment and instrumentation were provided by the North Sanpete High School football program.

\section{Procedures}

All training sessions and 1-RM assessments took place at the North Sanpete High School Fitness Center and field assessments took place in the North Sanpete High School gymnasium. Both facilities being located in Mt. Pleasant, Utah.

Participants were given verbal instruction on procedures and proper lifting and power-assessment protocols. They then underwent a pre-assessment of their weight room performance in the 1-RM BS and PC as well as with their field tests involving the VJ and 9.14 meter sprint. Their pre-assessment took place over a two day period. On day 1 field assessments were performed on the 9.14 meter sprint and the VJ. Prior to initiating the field tests the participants warmed up by completing a 400 meter jog followed by a 10 minute dynamic warm-up over 28.65 meters. The dynamic warm-up included the following: high knees, butt kickers, A-skips, B-skips, back pedal in both directions, $75 \%$ track start, and $100 \%$ track start.

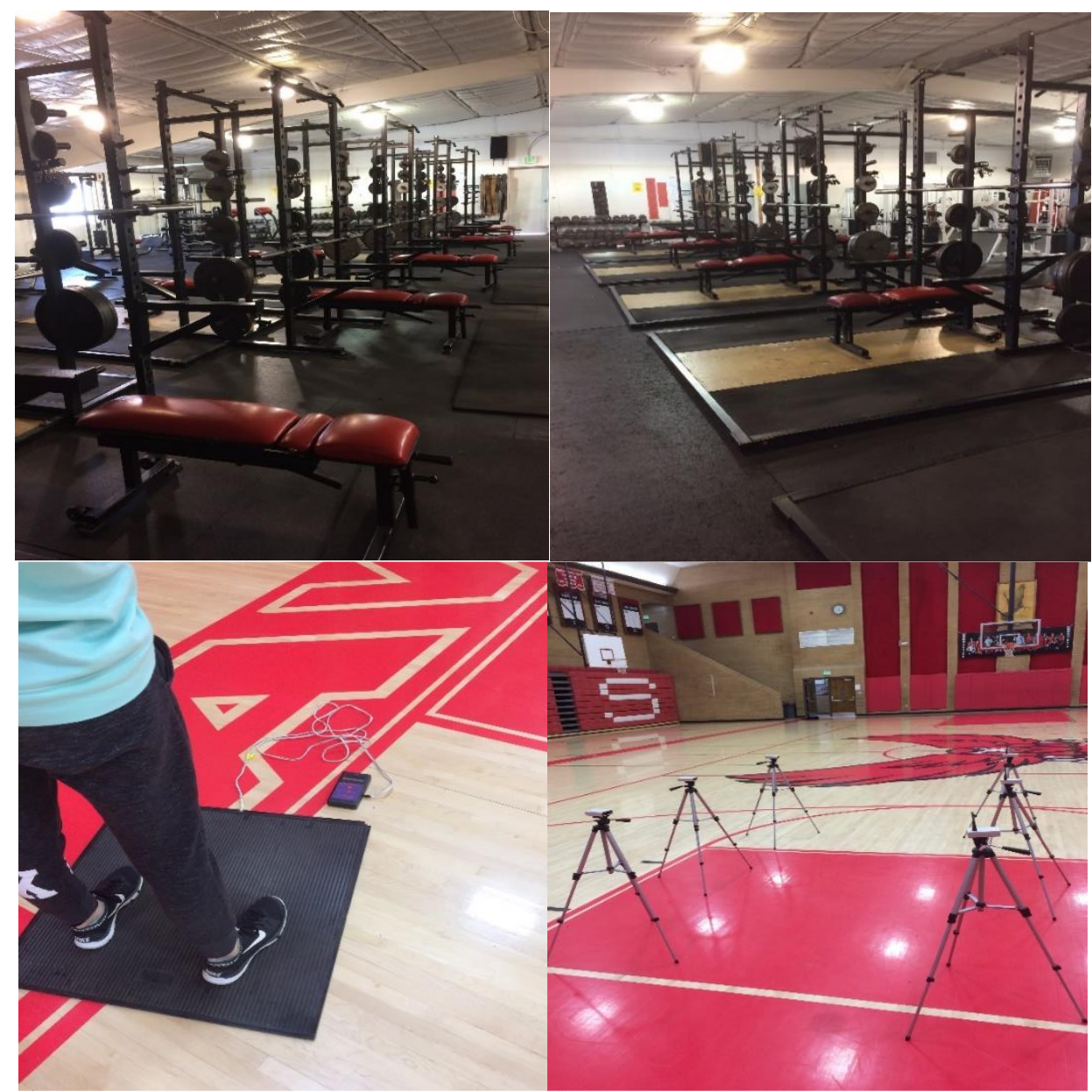

Figure 1. North Sanpete High School training facility and gymnasium located in Mt. Pleasant, Utah. 


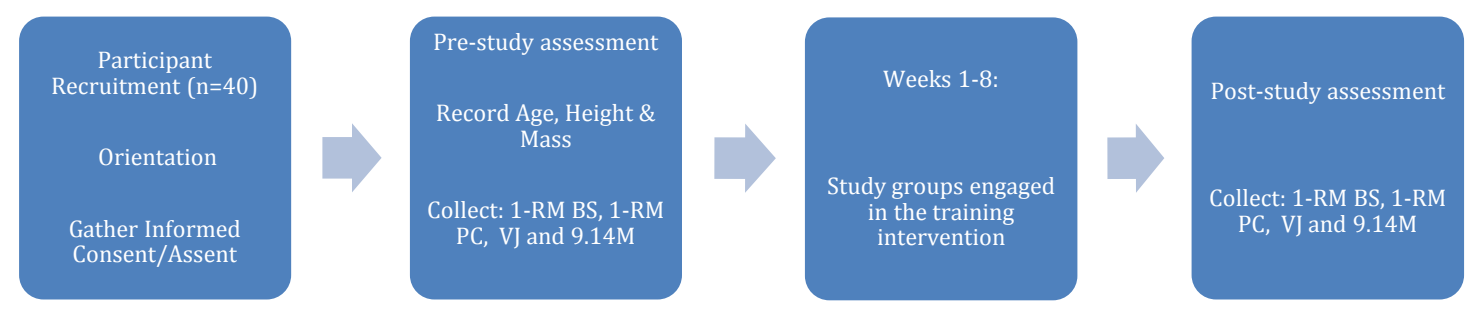

Figure 2. Study time line (BS-back squat; PC-power clean; 1-RM-one repetition maximum; VJ-vertical jump; 9.14M-meter sprint).

After the dynamic warm-up the participants were given proper instruction and demonstration of the 9.14 meter sprint using the Polaris Sports Timing System. The participants were required to complete at least 3 attempts and were allowed to continue until their time showed no improvement. Their fastest time was recorded. A three minute rest interval was required between each attempt. For the VJ again athletes were given instruction and demonstration of the proper jumping technique recommended by Probotics Just Jump system. The participants were required to complete at least 3 attempts and were allowed to continue until their height marks showed no improvement. Their highest jump was recorded. A three minute rest interval was required between each attempt. On day 2 weight room assessments were performed on the 1-RM BS and PC. The same warm-up procedures as previously mentioned were used prior to testing. Both lifting techniques were explained and demonstrated in order to achieve proper technique and safety. Participants were then instructed to perform a light warm-up set. They were then able to progress in weight until a failed attempt occurred, which then ended their assessment. The previous successful attempt was recorded as their 1-RM. A two to five minute rest interval was required between attempts. Their group placement was then determined by their 1-RM BS and PC pre-assessment and required a stratified random process. The participant's 1-RM BS and PC scores were added together for each individual and then the combined scores were ranked from highest to lowest. The participants were then randomly assigned in pairs to the TR and the FQ experimental groups. Specifically, the individuals with the highest two combined scores were randomly assigned to the experimental groups. Next, the individuals with the $3^{\text {rd }}$ and $4^{\text {th }}$ highest two combined scores were randomly assigned to the experimental groups. This process continued until both experimental groups were fully formed. This allowed the groups to be essentially equal for muscular strength and power as assessed by the dependent variables.

The FQ group's workout required athletes to perform a series of Olympic lifting derivatives over the course of a week which was comprised of: a power clean once a week and a hang clean progression workout twice a week. The first quarter of the Four Quarters workout was done by performing a high pull from power position in the hang clean, the second quarter involved performing a hang clean from the hang power position, the third quarter was performed by doing a hang clean followed by a power press, and the fourth quarter required performing a hang clean followed by a front parallel squat which concluded with a power press. The TR group's workout performed a dead lift three times a week. All other core lifts, such as the bench press, squat, other auxiliary lifts, and exercises were performed by both groups, which helped to eliminate any inconsistencies and established validity to the study.

The frequency of the training took place four days a week (Monday, Tuesday, Thursday, and Friday) with each session lasting 60 minutes. This training was part of their regular off-season strength and conditioning program. This program required participants to first complete a core lift using an ascending and descending pyramid consisting of seven sets with the following periodization weight load (repetitions $\times \%$ 1-RM load): $15 \times 30 \%, 8 \times 50 \%, 5 \times 65 \%, 3 \times 80 \%, 1 \times 100 \%, 8 \times 65 \%$, $10 \times 50 \%$. Progression was based off the 1-RM preassessments. The participants had to be able to successfully complete the required repetitions before they could increase their resistance by $2.3 \mathrm{~kg}$ ( 5 pounds) for the next week. The only variation to their 
lifting program was the prescribed Olympic lifts derivatives or dead lifts. The FQ and TR groups completed the following weight periodization load (sets $\mathrm{x}$ repetitions $\mathrm{x} \%$ 1-RM load): Week 1, 3x5x60\%,

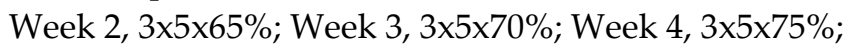

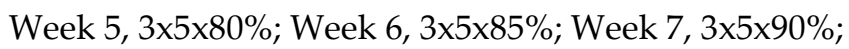
Week $8,3 \times 5 \times 95 \%$. Participants were also required to complete auxiliary lifts focusing on different muscle groups on different days completing 3 sets of 8 repetitions for each lift and a daily abdominal resistance program with 3 sets of 15 repetitions. Tables 1 and 2 give a more detailed explanation of the participants' weekly training programs. Any participant that missed a session was required to make up the workout. The duration of the resistance training intervention took place over eight weeks. At the end of that time, they completed a post-assessment, which included all of the same pre-assessment tests. All data was recorded on both data analysis sheets and stored in a computer for further evaluation.

\section{Reliability}

All descriptive and dependent variables were collected as described by Tricoli, Lamas, Carnevale, \& Urginowitsch (2005) and Sierer et al (2008). The validity for the dependent variables of 1-RM BS and 1-RM PC were established in a study by Cormie et al., (2007). High reliability of electronic timing systems for the 9.14 meter sprint has been demonstrated by Nikolenko et al. (2011). The VJ test was performed using the techniques from the Brown \& Weir (2001) study that noted a high reliability for the $\mathrm{VJ}$.

Table 1

Four quarters (FQ) weekly strength training program.

\begin{tabular}{cccc}
\hline Monday & Tuesday & Thursday & Friday \\
\hline Squat & Bench & Power Clean & Squat \\
Four Quarters* & Incline & Lat. Pull Down & Four Quarters* \\
Leg Extensions & Decline & Cable Rows & EZ Bar Bicep Curls \\
Leg Curls & Fly's & Superman's & Hammer Curls \\
Calf Raises & Skull Crushers & Shoulder Raises & Spider Curls \\
Abdominals & Abdominals & Abdominals & Abdominals \\
\hline $\begin{array}{l}\text { Note: Core Lifts (Squat, Bench, } \mathcal{E} \text { Power Clean); FQ (Mondays \& Fridays); Auxiliary Lifts } \\
\text { (Monday Leg Aux., Tuesday Chest } \mathcal{E} \text { Triceps Aux., Thursday Back \& Deltoid Aux., Friday Bicep } \\
\text { Aux.); Abdominals done every day. }\end{array}$ &
\end{tabular}

Table 2

Traditional resistance (TR) weekly strength training program.

\begin{tabular}{|c|c|c|c|}
\hline Monday & Tuesday & Thursday & Friday \\
\hline Squat & Bench & Dead Lift & Squat \\
\hline Dead Lift* & Incline & Lat. Pull Down & Dead Lift* \\
\hline Leg Extensions & Decline & Cable Rows & EZ Bar Bicep Curls \\
\hline Leg Curls & Fly's & Superman's & Hammer Curls \\
\hline Calf Raises & Skull Crushers & Shoulder Raises & Spider Curls \\
\hline Abdominals & Abdominals & Abdominals & Abdominals \\
\hline
\end{tabular}




\section{Design and Analysis}

The dependent variable pre- and post-assessment scores were obtained for the 1-RM BS, 1-RM PC, VJ and 9.14 meter sprint. Pre- post- scores were compared with-in each group with a dependent t-test. A gain score was also calculated for each dependent variable (post-pre score) and compared between the experimental groups (FQ vs TR) with an independent t-tests $(p<0.05)$.

\section{Results}

There were 40 high school male football players between 14 to 18 years old that participated in this study. Twenty participants were in the FQ group and 20 were in the TR group. All participants completed this study and their results have been recorded, reported, analyzed, and included in the study. Descriptive participant information can be found in Table 3 that summarizes the mean and standard deviations of both groups.

Both the TR and FQ groups made improvements in pre to post assessments in the 1-RM BS, 1-RM PC, and
VJ ( $p<0.05)$. The gain scores for the 1-RM BS, 1-RM PC were significantly greater for the FQ group $(p<0.05)$. Neither group improved 9.14 meter sprint times ( $p>0.05)$. A summary, including the mean and standard deviation, of the dependent variables pre, post and gain scores can be found in tables 4 and 5 .

\section{Discussion}

The purpose of this study was to determine the effects of Olympic style lifting, more specifically the Four Quarters work-out, on speed, strength, and power in comparison to traditional lifting on adolescent, male, North American High School football players. In order to do so two experimental groups were formed and completed the same resistance training program, however, the only variation that existed is one group (FQ) performed a progression of Olympic lift derivatives and the second group (TR) performed a dead lift twice a week. It was hypothesized that the utilization of the Four Quarters RT protocol would yield greater speed, power and strength gains than those using a TR protocol.

Table 3

Participant descriptive information (Mean \pm SD).

\begin{tabular}{lcccc}
\hline Variables & $\mathrm{N}$ & Age (years) & Height $(\mathrm{cm})$ & Mass $(\mathrm{kg})$ \\
\hline Olympic Lifters (FQ) & 20 & $15.8 \pm 0.8$ & $180.5 \pm 6.08$ & $79.2 \pm 16.5$ \\
Traditional Resistance (TR) & 20 & $16.0 \pm 0.9$ & $180.2 \pm 6.87$ & $77.4 \pm 18.7$ \\
\hline
\end{tabular}

Table 4

Olympic lifting (FQ) and traditional resistance (TR).

\begin{tabular}{ccccccc}
\hline & \multicolumn{3}{c}{ 1-RM Back Squat $(\mathrm{kg})$} & \multicolumn{3}{c}{ 1-RM Power Clean $(\mathrm{kg})$} \\
\cline { 2 - 6 } & Pre & Post & Gain & Pre & Post & Gain \\
\hline FQ & $98.9 \pm 29.9$ & $115.9 \pm 29.9^{*}$ & $17.0 \pm 8.0^{*}$ & $70.9 \pm 18.2$ & $82.3 \pm 16.7^{*}$ & $11.4 \pm 4.8^{*}$ \\
TR & $97.2 \pm 29.5$ & $107.5 \pm 24.2^{*}$ & $10.3 \pm 11.7$ & $70.7 \pm 15.6$ & $76.7 \pm 15.3^{*}$ & $6.0 \pm 4.0$ \\
\hline
\end{tabular}

Note: Participant means and standard deviations for dependent variables. ${ }^{*}$ Significant improvement pre to post intervention $p<0.05$.

${ }^{¥}$ Significant improvement difference in gain scores $p<0.05$.

Table 5

Olympic lifting (FQ) and traditional resistance (TR).

\begin{tabular}{ccccccc}
\hline & \multicolumn{3}{c}{ Vertical Jump $(\mathrm{cm})$} & \multicolumn{3}{c}{9.14 Meter Sprint (sec) } \\
\cline { 2 - 6 } & Pre & Post & Gain & Pre & Post & Gain \\
\hline FQ & $52.4 \pm 8.97$ & $55.2 \pm 7.03^{*}$ & $2.9 \pm 3.94$ & $1.97 \pm 0.15$ & $1.94 \pm 0.10$ & $-0.03 \pm 0.10$ \\
TR & $51.3 \pm 7.88$ & $53.9 \pm 7.51^{*}$ & $2.6 \pm 4.21$ & $1.96 \pm 0.18$ & $1.98 \pm 0.13$ & $0.01 \pm 0.12$ \\
\hline
\end{tabular}

Note: Participant means and standard deviations for dependent variables. *Significant improvement pre to post intervention $p<0.05$. 
The results of the study revealed that both the TR and FQ groups made improvements in pre to post assessments in the 1-RM BS, 1-RM PC, and VJ ( $\mathrm{p}<0.05)$, and the gain scores for the 1-RM BS, 1-RM PC were significantly greater for the FQ group $(p<0.05)$. Neither group improved 9.14 meter sprint times $(p>0.05)$.

The mean initial assessment VJ scores for the two experimental groups ranged from 51.3-52.4 cm which is approximately $40^{\text {th } \% \text { ile }} \mathrm{VJ}$ scores for $10^{\text {th }}$ grade male North American football players (Hoffman, 2007). The post assessment mean 1-RM BS scores for both groups was $112.0 \mathrm{~kg}$ which is comparable to $25^{\text {th\%ile }} 1-\mathrm{RM}$ BS scores for 14-15 year old male North American football players (Hoffman, 2007). The post assessment mean 1RM PC scores for both groups was $79.5 \mathrm{~kg}$ which is comparable to $50^{\text {th\%ile }} 1$-RM PC scores for $14-15$ year old male North American football players (Hoffman, 2007). The 1-RM BS/body mass ratios for the FQ and TR were 1.25 and 1.26 respectively, which were slightly lower than those reported by Thompson et al. (2017) for male high school athletes ( $\approx 1.64$ 1-RM BS/body mass). The mean 9.14 meter sprint times ranged from 1.94-1.98 seconds and were as expected, slower than those reported by Springall et al. (2016) for collegiate track athletes $(1.68 \pm 0.14 \mathrm{sec})$.

The findings of this study in regards to improvement in pre and post-assessment tests (1-RM squat, 1-RM power clean, and VJ) are consistent with the following previously conducted studies that demonstrated strength and power gains in children and adolescents that participated in RT programs (Faigenbaum et al., 2007; Faigenbaum et al., 1996). Through their findings it was determined that young athletes can develop increased power and strength by participating in resistance RT programs. Other studies by Hackett et al., (2016) and Hoffman et al., (2004) demonstrated increased VJ height in young athletes after implementing an Olympic weightlifting program, which is also consistent with the results of this study. The effect size (ES) for the 1-RM BS strength gains for the FQ group was 0.57 and for the TR group it was 0.35 . The ES in 1-RM BS strength gains for both groups was reasonably consistent with three meta-analyses regarding strength gains in youths following a RT program (Behringer et al., 2010; Faigenbaum, 1996; Payne et al., 1997).

The findings in this study also demonstrated significant improvement for the FQ group in the 1-RM squat and 1-RM power clean which were consistent with studies by Ebben \& Blackard (1997) and Burger et al. (2000), however, these studies also included complex and plyometric exercises combined with Olympic lifting to achieve their results. Another study by McBride et al. (1999) compared Olympic lifting to traditional lifting in a sample of sprinters and found that Olympic lifters were significantly stronger and produced more force than participants in the experimental groups that did include Olympic lifts. McBride et al.'s (1999) findings were consistent with the results of the current study in that they found that Olympic lifting elicited more strength and power gains when compared to traditional lifting. Another study by Harbili \& Alptekin (2014) found that performing a progression of Olympic lifts, such as the FQ RT program used in the current study, can assist in sustaining power output after the first repetition.

The results of the current study for the 9.14 meter sprint were not significant, which was inconsistent with other studies. For example, the results from the Peterson et al. (2006) and Chaouachi et al. (2014) studies found that increased power, particularly power improvements as a result of performing the Olympic lifts, correlated to improvements in running speed. However, their increased speed gains could be accredited to their use of plyometric and running drills. These studies also used longer distances (20 to 36.6 meters) for assessment as compared to the 9.14 meter sprint distance assesses in the current study.

This study implemented the FQ training protocol that focused on progressions of Olympic lifting derivatives that at times included the catch position. Previous work by Sanders et al. (2017) has indicated that Olympic lifts derivatives not requiring the catch are just as beneficial (i.e. strength and power improvements) as derivatives requiring the catch. Sanders et al. (in review) suggested that the performance increases noted in their study were more related to the "completion of the explosive triple extension than to the catch". It should be noted that receiving the bar in the catch position may lead to possible increased risk of injury and joint trauma (Suchomel et al., 2015). As such, Sanders at al. (2017) and Suchomel et al. (2015) suggested that "it would be prudent for coaches to not employ the catch during most, if not all repetitions of snatch and hang derivatives". We agree with Sanders at al. (2017) and Suchomel et al. (2015) in suggesting that future studies 
should continue to explore the benefits of the varying Olympic lifting derivatives.

There were primary three limitations to this study. The main limitation was the age of the athletes. They ranged in ages from 14-18 years old. Because of this, instances occurred in which the athletes couldn't get rides from their parents, they would sleep in, or they weren't as dedicated or motivated to the strict adherence of the study. Despite the fact that they all completed the program, there could be inconsistencies in performance due to the work-out time difference from the morning to the make-up session. With that being said, they were on average all able to achieve strength and power gains in the 1-RM BS, 1-RM PC, and VJ.

Another limitation could come from the experience of the athletes. Most of the subjects in the study could be considered novice lifters. Due to the lack of experience, strength gains were inevitable. This may have skewed the final results when it came to the outcomes related to strength. Regardless of this, strength and power gains were made with both the FQ and TR group. Further, the randomized process of establishing the experimental groups should have minimized the extent of any impact on internal validity.

Finally another limitation in the study could be length of the 9.14 meter sprint. Due to inclement outdoor weather conditions and the lack of available space at the indoor facility it was impossible to perform more than just a 9.14 meter sprint. It is possible that significant gains in running speed may have been noted if the participants had completed a longer sprint (36.6 meter sprint) as the assessment of speed.

Future research still needs to be conducted in order to see if strength and power gains in the weight room vary between Olympic power lifters and traditional weight lifters in high school athletic programs, especially since the experimental and clinical data is limited in this population. If may be of benefit to research varsity athletes due to the fact that they have more experience lifting, which may give more valid and reliable findings.

\section{Conclusion}

This study demonstrated that the two types of RT protocols were easily implemented and lead to significant improvements in the 1-RM BS, 1-RM PC and VJ in High School North American Football players. However, RT protocols that integrate Olympic lift derivatives, can lead to superior gains in strength and power when compared to TR protocols. Finally, strength and conditioning specialists should include sprint specific drills to the RT protocols used in this study as the development of running speed is typically a goal of any strength and conditioning program.

\section{Conflict of Interest Declaration}

No funding was received for this research. The authors have no conflict of interest related to this research.

\section{References}

Arnheim DD, Prentice WE. Principles of Athletic Training (10 $10^{\text {th }}$ ed.). Boston, Massachusetts: McGraw Hill, 2000.

Behringer M, vom Heede A, Yue Z, Mester J. Effects of resistance training in children and adolescents: a meta-analysis. Pediatrics, 2010; 126(5): e1199-1210.

Brown LE, Weir JP. ASEP procedures recommendation I: Accurate assessment of muscular strength and power. JEP Online, 2001; 4(3): 1-21.

Bruce-Low S, Smith D. Explosive exercises in sports training: A critical review. JEP Online, 2007; 10(1): 21-33.

Burger T, Boyer-Kendrick T, Dolny D. Complex training compared to a combined weight training and plyometric training program. J Strength Cond Res, 2000; 14(3): 360.

Chaouachi A, Hammami R, Kaabi S, Chamari K, Drinkwater EJ, Behm DG. Olympic weightlifting and plyometric training with children provides similar or greater performance improvements than traditional resistance training. J Strength Cond Res, 2013; 28(6): 1483-1496.

Channell BT, Barfield JP. Effect of Olympic and traditional resistance training on vertical jump improvement in high school boys. J Strength Cond Res, 2008; 22(5): 1522-1527.

Cormie P, McBride JM, McCaulley GO. Validation of power measurement techniques in dynamic lower body resistance exercises. J Appl Biomech, 2007; 23(2): 103-118.

Dahab KS, McCambridge TM. Strength training in children and adolescents: raising the bar for young athletes? Sports Health, 2009; 1(3): 223-226.

Ebben WP, Blackard DO. Complex training with combined explosive weight training and plyometric exercises. Olympic Coach, 1997; 7(4): 11-12.

Enoka RM. Neuromechanics of Human Movement (3 ${ }^{\text {rd }}$ ed.). Champaign, IL: Human Kinetics, 2002.

Faigenbaum AD, McFarland JE, Keiper FB, Tevlin W, Ratamess NA, Kang J, Hoffman JR. Effects of a short-term plyometric and 
resistance training program on fitness performance in boys age 12 to 15 years. J Sport Sci Med, 2007; 6: 519-525.

Faigenbaum AD, Kraemer WJ, Cahill B, Chandler J, Dziados J, Elfrink LD, Forman E, Gaudiose M, Micheli L, Nitka M, Roberts S. Youth resistance training: Position statement paper and literature review. Strength Cond J, 1996; 18(6): 6275.

Faigenbaum AD, McFarland JE, Keiper FB, Tevlin W, Ratamess NA, Kang J, Falk B, Tenenbaum G. The effectiveness of resistance training in children. A meta-analysis. Sports Medicine, 1996; 22(3): 176-186.

Fore C. Building Championship-Caliber Football Programs: Inside the Locker Rooms and Minds of State Champion Head Football Coaches. Monterey, CA: Coaches Choice, 2013.

Frounfelter G. Triple extension: the key to athletic power. NSCA's Performance Training Journal, 2009; 8(1): 14-15.

Garhammer J. A review of power output studies of Olympic and powerlifting: methodology, performance prediction, and evaluation tests. J Strength Cond Res, 1993; 7(2): 76-89.

Greenberg N. Von Miller is going to get paid after Super Bowl 50 win. The Washington Post, February 8, 2016. Retrieved from https://www.washingtonpost.com/news/fancystats/wp/2016/02/08/von-miller-is-going-to-get-paid-aftersuper-bowl-50-win/?utm_term=.ec524d44ea79

Hackett D, Davies T, Soomro N, Halaki M. Olympic weightlifting training improves vertical jump height in sportspeople: a systematic review with meta-analysis. Br J Sports Med, 2016; 50(14): 865-872.

Haff GC, Whitley A, Potteiger JA. A brief review: Explosive exercises in sports performance. Strength Cond J, 2001; 23(3): 13-20.

Harbili E, Alptekin A. Comparative kinematic analysis of the snatch lifts in elite male adolescent weightlifters. J Sport Sci Med, 2014; 13: 417-422.

Hendrick A, Wada H. Weightlifting movements: do the benefits outweigh the risks? Strength Cond J, 2008; 30(6): 26-35.

Hoffman JR. Effects of a short-term plyometric and resistance training program on fitness performance on boys age 12-15 years. J Sport Sci Med, 2007; 6, 519-525.

Hoffman JR, Cooper J, Wendell M, Kang J. Comparison of Olympic vs. traditional power lifting training programs in football players. J Strength Cond Res, 2004; 18(1): 129-135.

McBride JM, Triplett-McBride T, Davie A, Newton RU. A comparison of strength and power characteristics between power lifters, Olympic lifters, and sprinters. J Strength Cond Res, 1999; 13(1): 58-66.
Nikolenko M, Brown LE, Coburn JW, Spiering BA, Tran TT. Relationship between core power and measures of sport performance. Kinesiology, 2011; 43(2): 163-168.

Patra K. Broncos LB Von Miller named Super Bowl 50 MVP. February 7, 2016. Retrieved from http://www.nfl.com./news/story/0ap3000000634374/article/ broncos-lb-von-miller-named-super-bowl-50-mvp

Payne VG, Morrow JJR, Johnson L, Dalton SN. Resistance training in children and youth: a meta-analysis. Research Quarterly for Exercise and Sport, 1997; 68(1): 80-88.

Peterson MD, Alvar BA, Rhea MR. The contribution of maximal force production to explosive movement among young collegiate athletes. J Strength Cond Res, 2006; 20(4): 867-873.

Sanders C, Sevene T, Adams KJ, DeBeliso M. A pilot comparison of the hang clean and hang snatch to the clean pull and snatch pull. J Athl Enhanc, 2017; 6: 5. doi: 10.4172/2324-9080.1000269

Sierer S, Battaglini CL, Mihalik JP, Shields EW, Tomasini NT. The national football league combine: Performance differences between drafted and non-drafted players entering the 2004 and 2005 drafts. J Strength Cond Res, 2008; 22(1): 6-12.

Small EW, McCambridge TM, Benjamin HJ, Bernhardt DT, Brenner JS, Cappetta CT, Congeni JA, Gregory AJM, Griesemer BA, Reed FE, Rice SG. Strength training by children and adolescents. Pediatrics, 2008; 121(4): 835-840.

Springall B, Larson A, DeBeliso M. The effects of a heavy resistance warm-up on sprint speed: A post activation potentiation study. Journal of Physical Education Research, 2016; 3(3): 5265.

Suchomel T, Comfort P, Stone M. Weightlifting pulling derivatives: rationale for implementation and application. Sports Med, 2015; 45(6): 823-839.

Thompson T, Berning J, Harris C, Adams KJ, DeBeliso M. The effects of complex training in male high school athletes on the back squat and vertical jump. International Journal of Sports Science, 2017; 7(2): 50-55.

Tricoli V, Lamas L, Carnevale R, Ugrinowitsch C. Short-term effects on lower-body functional power development: Weightlifting vs. vertical jump training programs. J Strength Cond Res, 2005; 19(2): 433-437.

Warning JT, Pfeiffer KA, Vorkapich M, Diltz A, Pivarnik JM. Efficacy of two resistance training modes in adolescents. Pediatric Exercise Science, 2016; 28: 34.

Weaver EK, DeBeliso M. Survey of Utah high school football strength and conditioning coaches. Journal of Sports Science, 2015; 3: $117-126$. 